ARTIGO ORIGINAL ORIGINAL ARTICLE

\section{Custo-minimização da troca entre imunoglobulinas de diferentes vias e marcas: sustentabilidade relacionada ao tratamento na saúde suplementar}

\author{
Cost minimizing the switching between \\ immunoglobulin of different routes and brands: of \\ treatment-related in the supplementary health
}

Harli Pasquini Netto', Yohanna Ramires', Bianca Fontana Aguiar², Jolline Lind³, Priscila Del Pilar Arriagada Gajardo ${ }^{4}$, Moacir Pires Ramos 5 , Jaime Luis Lopes Rocha ${ }^{6}$

DOI: $10.21115 / J B E S . v 12 . n 1 . p 32-8$

\begin{abstract}
Palavras-chave:
custo-minimização,

imunoglobulina $\mathrm{G}$, administração intravenosa, administração subcutânea, saúde suplementar medicina baseada em evidências
\end{abstract}

\section{Keywords:}

cost-minimization, immunoglobulin G, intravenous administration, subcutaneous administration, supplementar health, evidence-based medicine

\section{RESUMO}

Objetivo: Avaliar custo-minimização da troca entre as versões intravenosa (IVlg) e subcutânea (SClg) das imunoglobulinas (lg) em operadora de saúde com mais de 500.000 vidas. Métodos: Estudo retrospectivo, transversal, descritivo, seguido de custo-minimização entre os pacientes que utilizaram IVlg, de $1^{\circ}$ de outubro de 2018 a 30 de setembro de 2019. Simulou-se a troca entre as IVlg e SClg, objetivando descrever a economia de uma hipotética substituição. Estabeleceram-se como critérios de exclusão: o não pagamento e a liberação com dose acima de 60.000 mg. Após exclusão, calcularam-se as despesas totais, somando-se os custos do produto e taxas de infusão. Resultados: Evidenciou-se que 133 pacientes, totalizando 1.175 liberações, utilizaram IVlg no período avaliado. Identificou-se a utilização de 34.797 .500 mg de IVIg, por 10 especialidades, totalizando R\$ 12.408.192,50 de despesas. Quando aplicada simulação, há uma potencial economia de recursos de até 29,83\%, dependendo da SClg escolhida. Conclusão: A análise econômica no tratamento com imunoglobulinas evidenciou significativa relevância, pois contribui com o uso adequado da terapêutica garantindo a sustentabilidade do sistema de saúde. Medicamentos subcutâneos apresentam-se como uma opção custo-minimizatória em comparação ao tratamento intravenoso para saúde suplementar brasileira.

\section{ABSTRACT}

Objective: Cost-minimization evaluation of the switch from intravenous (IVIg) to subcutaneous (SClg) immunoglobulin (lg) in a Brazilian Health Maintenance Organization (HMO), with more than 500.000 lives. Methods: This is a retrospective, transversal and descriptive study, followed by a cost-minimization analysis among patients using IVlg between 2018, October, $1^{\text {st }}$ and 2019, September, $30^{\text {th }}$. The simulation was performed supposing the exchange from IVIg to SClg, in order to calculate possible savings. Exclusion criteria: non-payment (gloss), and infusions with doses above 60.000 miligrams. After exclusion, total expenditures were calculated by summing product and infusion costs. Results: There were133 patients, with1,175 IVIg infusion events in the period evaluated. It was identified the use of 34,797,500 milligrams of IVIg, for 10 specialties, with $\mathrm{R} \$ 12,408,192.50$ of final expenditure. The simulation previews hypothetical reduction in the final cost of up to $29.83 \%$, depending on the SClg brand chosen. Conclusion: The economic analysis in the treatment with

\footnotetext{
Recebido em: 14/01/2020. Aprovado para publicação em: 21/03/2020.

1. Mestre em Ciências Farmacêuticas, Centro de Pesquisa e Inovação, Unimed Curitiba, Curitiba, PR, Brasil.

2. Especialista em Saúde da Criança e do Adolescente, Centro de Pesquisa e Inovação, Unimed Curitiba, Curitiba, PR, Brasil.

3. Mestre em Enfermagem, Centro de Pesquisa e Inovação, Unimed Curitiba, Curitiba, PR, Brasil.

4. Especialista em Estatística com Ênfase em Pesquisa, Planejamento Estratégico, Unimed Curitiba, Curitiba, PR, Brasil.

5. Epidemiologista, Centro de Pesquisa e Inovação, Unimed Curitiba, Curitiba, PR, Brasil.

6. Infectologista. Professor de Infectologia da Pontifícia Universidade Católica do Paraná. Centro de Pesquisa e Inovação, Unimed

Curitiba, Curitiba, PR, Brasil.

Instituição onde o trabalho foi executado: Centro de Pesquisa e Inovação, Unimed Curitiba, Curitiba, PR, Brasil.

Financiamento: Não houve.

Congressos: VI Fórum Brasileiro de Assistência Farmacêutica, 2019.

Autor correspondente: Harli Pasquini Netto. Avenida Affonso Penna, 297, Tarumã, Curitiba, PR, Brasil. Telefone: (42) 99920-6968 E-mail: netto_pasquini@hotmail.com
} 
immunoglobulins showed significant relevance, as it contributes to the appropriate use of therapy ensuring the sustainability of the health system. Subcutaneous drugs are a cost-minimizing option compared to intravenous treatment for Brazilian HMOs.

\section{Introdução}

Com número estimado de aproximadamente 6 milhões de pacientes diagnosticados no mundo todo (Bousfiha et al., 2013), os distúrbios de imunodeficiência são condições causadas por defeitos no sistema imunológico que deixam o corpo incapaz de produzir anticorpos suficientes ou de qualidade para o combate de doenças infecciosas, entre outras funções. Tais distúrbios são classificados em primários ou secundários de acordo com a sua origem, sendo os primários aqueles defeitos habitualmente congênitos e crônicos no sistema imunológico adaptativo ou inato. Já os defeitos secundários são aqueles adquiridos por meio da exposição a um agente externo, como infecção, quimioterapia, desnutrição ou queimaduras graves, habitualmente com manifestação temporária (Bonilla et al., 2015; Picard et al., 2015).

Essa doença pode afetar um ou mais componentes do sistema imunológico, incluindo células B humorais, células T ou combinadas, além de distúrbios das células fagocitárias e atuação de intercooperação na disfunção proteica (International Union of Immunological Societies Expert Committee on Primary Immunodeficiencies, 2009). As imunodeficiências primárias (IDPs) mais comuns são as condições de deficiência de anticorpos, como a imunodeficiência comum variável, a agamaglobulinemia ligada ao $\mathrm{X}$ e deficiências específicas de anticorpos, como a deficiência de lgA, entre outras (Perez et al., 2017).

O tratamento para IDPs varia de acordo com o tipo do distúrbio, no entanto, salvo a exceção da deficiência seletiva de $\operatorname{lgA}$, a terapia de reposição de IG é o tratamento de primeira escolha para condições de deficiência de anticorpos (Perez et al., 2017). A terapia de reposição de IG pode ser administrada via infusão intravenosa (IVIg) ou via infusão subcutânea de IG (SClg), sendo ambas já comprovadamente eficazes e seguras (Högy et al., 2005; Shabaninejad et al., 2016), ademais, as SClg reduzem menos a qualidade de vida aos pacientes (Hadden \& Marreno, 2015; Igarashi et al., 2014).

Ressalta-se que o potencial econômico para a saúde pelo uso da SClg também é apontado por vários estudos europeus (Beauté et al., 2010; Högy et al., 2005; Lazzaro et al., 2014; Pollock \& Meckley, 2018) e um japonês (Igarashi et al., 2014). Devido à escassez de recursos e ao patamar de inflação constatado no mercado de saúde, tanto público quanto privado (Garcia et al., 2013), uma análise de custo-minimização na saúde suplementar brasileira mostra-se necessária.

Neste estudo, objetivou-se avaliar o impacto financeiro, a partir de uma análise simulada de custo-minimização da troca entre diferentes apresentações de imunoglobulinas (lgs) em uma operadora de plano de saúde responsável por mais de 500.000 vidas.

\section{Métodos}

\section{Características gerais do estudo}

O presente estudo visa a uma simulação retrospectiva transversal e descritiva dos custos de uma operadora de planos de saúde com mais de 500.000 vidas no sul do Brasil, por meio de análises de custo-minimização. Como objetivo primário, busca-se uma projeção da potencial economia gerada pela substituição das imunoglobulinas intravenosas (IVlg) pelas subcutâneas ( $\mathrm{SClg}$ ) no tratamento de diferentes patologias.

Para tanto, uma análise de custo-minimização com base em um modelo de decisão foi desenvolvida. Esse modelo de análise determinística constitui-se na avaliação econômica que simula diferentes alternativas em relação ao potencial custo evitado por ocasião da substituição entre as apresentações IVIg para SClg (Beauté et al., 2010; Chapel et al., 2000; Drummond et al., 2005; Gardulf et al., 1995). Para que essa análise seja possível, devem-se considerar eficácia e segurança similares entre IVIg e IVIg (Beauté et al., 2010; Chapel et al., 2000; Gardulf et al., 1995; Shabaninejad et al., 2017), portanto passível de troca médica em diversas situações clínicas (Hadden \& Marreno, 2015; Shabaninejad et al., 2016). As diferentes apresentações das imunoglobulinas foram escolhidas para este estudo devido à grande variabilidade em seus preços.

\section{Características do banco de dados, da busca, da extração e da população do estudo}

A população-alvo do presente estudo foi composta por pacientes que efetivamente fizeram uso das diferentes marcas de IVIg (Endobulin Kiovig; Flebogamma` e Sandoglobulina Privigen ${ }^{\circ}$ no período de $1^{\circ}$ de outubro de 2018 a 30 de setembro de 2019, na rede prestadora de uma única operadora de plano de saúde, independentemente da indicação médica. Projetou-se a possível permuta entre o medicamento IVlg e SClg. Foram considerados como critérios de exclusão o não pagamento por critérios de auditoria e doses acima de 60.000 mg por liberação, o que corresponde a um volume excessivo para infusão mensal de apresentações SClg (Endobulin Kiovig 100 mg/mL: 600 mL, Gammanorm 165 mg/mL: 363 mL, Hizentra`200 mg/mL: 300 mL e Hyqvia' 100 mg/mL: 600 mL).

A população foi identificada por meio do sistema informatizado exclusivo da operadora, sendo definidos como obje- 
tos de estudo somente dados econômicos e administrativos. Essa ferramenta de Business Intelligence (BI) permite a coleta de dados, organização, análise e monitoramento para a tomada de decisões assertivas, sendo a base para a identificação de oportunidades de investimentos, bem como para o controle do custo assistencial e sinistralidade da operadora de saúde.

Os dados importados da base informatizada foram organizados e armazenados em planilhas por meio do software Microsoft Excel', versão 2010. Os pacientes elegíveis ao estudo foram classificados conforme distribuição etária, sendo as faixas estipuladas pela raiz quadrada do número total da população e adequadas conforme o tamanho.

\section{Perspectiva da análise}

As análises foram realizadas na perspectiva da operadora de saúde suplementar, o que se aplica a todas as operadoras do país e podem ser adaptadas à perspectiva da saúde pública.

\section{Análise de custo-minimização}

Calcularam-se as despesas totais, somando-se os custos diretos com as apresentações das drogas, utilizando-se os preços-fábricas obtidos na lista da Câmara de Regulação do Mercado de Medicamentos (CMED) atualizada em 22 de janeiro de 2019 (Anvisa, 2019), acrescidos dos impostos regionais, que somam 18\%, além de taxa de infusão de medicamentos mediana praticada na saúde suplementar. Essas taxas de infusão foram referenciadas do estudo de Kashiura et al. (2018), que apresenta medianas de: taxa para infusão intravenosa no valor de $R \$ 103,50$ e subcutânea no valor de $\mathrm{R} \$ 9,00$. Para fins de anualização dos custos totais, foram utilizadas as frequências habitualmente aplicadas anualmente, ou seja: 13 aplicações para todas as IVlg; 52 aplicações para Endobulin Kiovig e Gammanorm ; 26 aplicações para Hizentra e 13 para Hyqvia.. Ressalta-se que, para pacientes com até 11 anos de idade, o cenário para o Hyqvia não foi aplicado por não haver essa indicação desse produto, portanto considerou-se a permanência do uso das IVlg.

\section{Resultados}

O relatório inicialmente gerado pelo sistema apresentou dados de mundo real, contabilizando 179 pacientes e totalizando 1.403 liberações de IVlg no período avaliado. Dessas liberações, 228 foram excluídas devido aos critérios de exclusão estipulados. Houve 208 liberações excluídas devido ao fato de o paciente receber mais de 60.000 mg por autorização (todos os casos para pacientes com idade $\geq 12$ anos). Além disso, excluíram-se mais 20 pacientes, por não pagamento em razão de critérios de auditoria. Foram três casos de pacientes com idade entre 0 e 11 anos e mais 17 casos com idade $\geq 12$ anos. Por fim, 1.175 liberações foram incluídas no estudo, totalizando um número populacional de 133 pacientes.
Dos 133 indivíduos incluídos, 74 eram do sexo feminino $(55,6 \%)$ e 59, do sexo masculino. Em relação à distribuição etária da população, foram estipuladas oito faixas: 0 a 11, 12 a 23, 24 a 35, 36 a 47, 48 a 59, 60 a 71, 72 a 83 e 84 a 95 anos, as quais apresentaram, respectivamente: 11, 8, 15, 22, 20, 20, 28 e 9 pacientes (Figura 1). A idade média e o desvio-padrão foram de 51,56 \pm 24,02 anos, com variação entre 0 e 87 anos de idade.

A análise identificou 1.175 liberações de IVlg elegíveis à intercambialidade das drogas, totalizando $34.797 .500 \mathrm{mg}$ de IVlg (13.377.500 mg de Endobulin Kiovig, 19.135 .000 mg de Flebogamma e $2.285 .000 \mathrm{mg}$ de Sandoglobulina ${ }^{\circ}$ ), utilizadas por 10 diferentes especialidades (Tabela 1). As análises de custos realizadas para a totalidade da população para as eventuais substituições de IVIg para SClg encontram-se representadas na Tabela 2.

A comparação do consumo anual das IVlg entre as especialidades, assim como os custos evitados totais dos cenários projetados para IVlg e SClg, mostrou-se expressivamente maior na Oncologia [consumo anual de 13.875 .000 mg (39,9\%) e custo total R\$5.016.504,50 (40,4\%)], Hematologia/Hemoterapia [consumo anual de $9.830 .000 \mathrm{mg}(28,2 \%)$ e custo total $\mathrm{R} \$ 3.500 .024,00(28,2 \%)]$, em uma proporção aproximada de 14 e 10 vezes o consumo e custos totais da especialidade Alergia e Imunoterapia, que apresentou um consumo anual de $1.110 .000 \mathrm{mg}$ (3,2\%) e custo total R\$386.394,00 (3,1\%) (Tabela 3).

O custo de tratamento de cada tecnologia foi composto pelo custo para aquisição das IVlg (Endobulin ${ }^{\circ}$ Kiovig: ${ }^{\circ}$ R\$ 0,344/ mg, Flebogamma : R\$ 0,360/mg e Sandoglobulina: $R \$ 0,300$ ) e SClg (Endobulin Kiovig: R\$ 0,344/mg, Gammanorm: R\$ 0,249/ mg, Hizentrå: R\$ 0,298/mg e Hyqvia:: R\$ 0,319/mg) obtidos na lista da CMED 2019 (Anvisa, 2019), acrescido do custo das taxas de infusão (Tabela 2).

Os resultados mostram que existe uma diferença importante entre os custos diretos das IVIg vs. SClg para o Flebogamma vs. (Endobulin Kiovig: -R\$ 0,016/mg, Gammanorm: -R\$ 0,111/mg, Hizentra:: -R\$ 0,062/mg e Hyqvia:: $-R \$ 0,041 /$

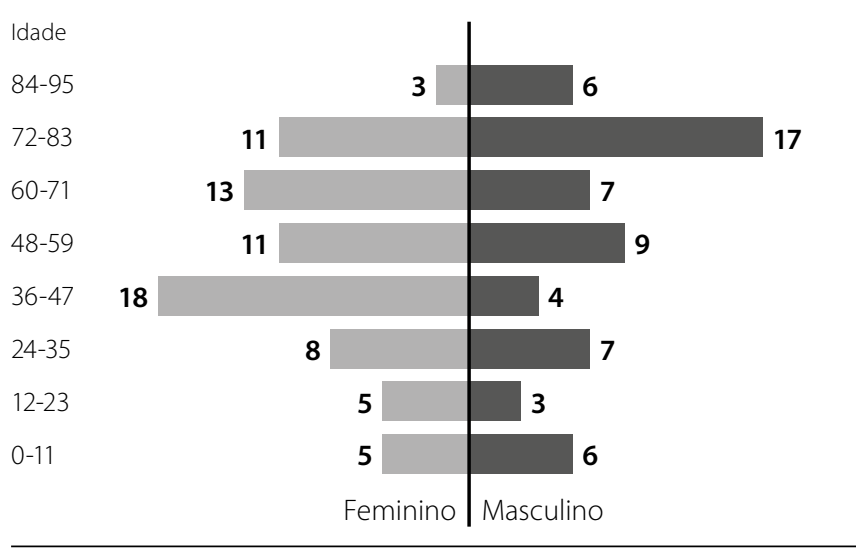

Figura 1. Distribuição de sexo e faixa etária dos pacientes incluídos no estudo. 
Tabela 1. Distribuição das liberações por faixa etária e especialidade médica, para o período de 12 meses

\begin{tabular}{|c|c|c|c|c|c|c|}
\hline \multirow[b]{2}{*}{ Especialidade/Idade } & \multicolumn{2}{|c|}{$\geq 12$ anos } & \multicolumn{2}{|c|}{$0-11$ anos } & \multicolumn{2}{|c|}{ Geral } \\
\hline & Liberações (N) & $\%$ & Liberações (N) & $\%$ & Liberações (N) & $\%$ \\
\hline Alergia e imunologia & 44 & $3,87 \%$ & - & - & 44 & $3,74 \%$ \\
\hline Hematologia e hemoterapia & 344 & $30,28 \%$ & - & - & 344 & $29,28 \%$ \\
\hline Clínica geral & 140 & $12,32 \%$ & - & - & 140 & $11,91 \%$ \\
\hline Neurologia & 57 & $5,02 \%$ & - & - & 57 & $4,85 \%$ \\
\hline Oncologia clínica & 375 & $33,01 \%$ & - & - & 375 & $31,91 \%$ \\
\hline Pediatria & 54 & $4,75 \%$ & 39 & $100 \%$ & 93 & $7,91 \%$ \\
\hline Pneumologia & 11 & $0,97 \%$ & - & - & 11 & $0,94 \%$ \\
\hline
\end{tabular}

Fonte: Elaborada pelos autores (2020).

Tabela 2. Custos diretos projetados para os tratamentos de IVIg e SClg, para 12 meses

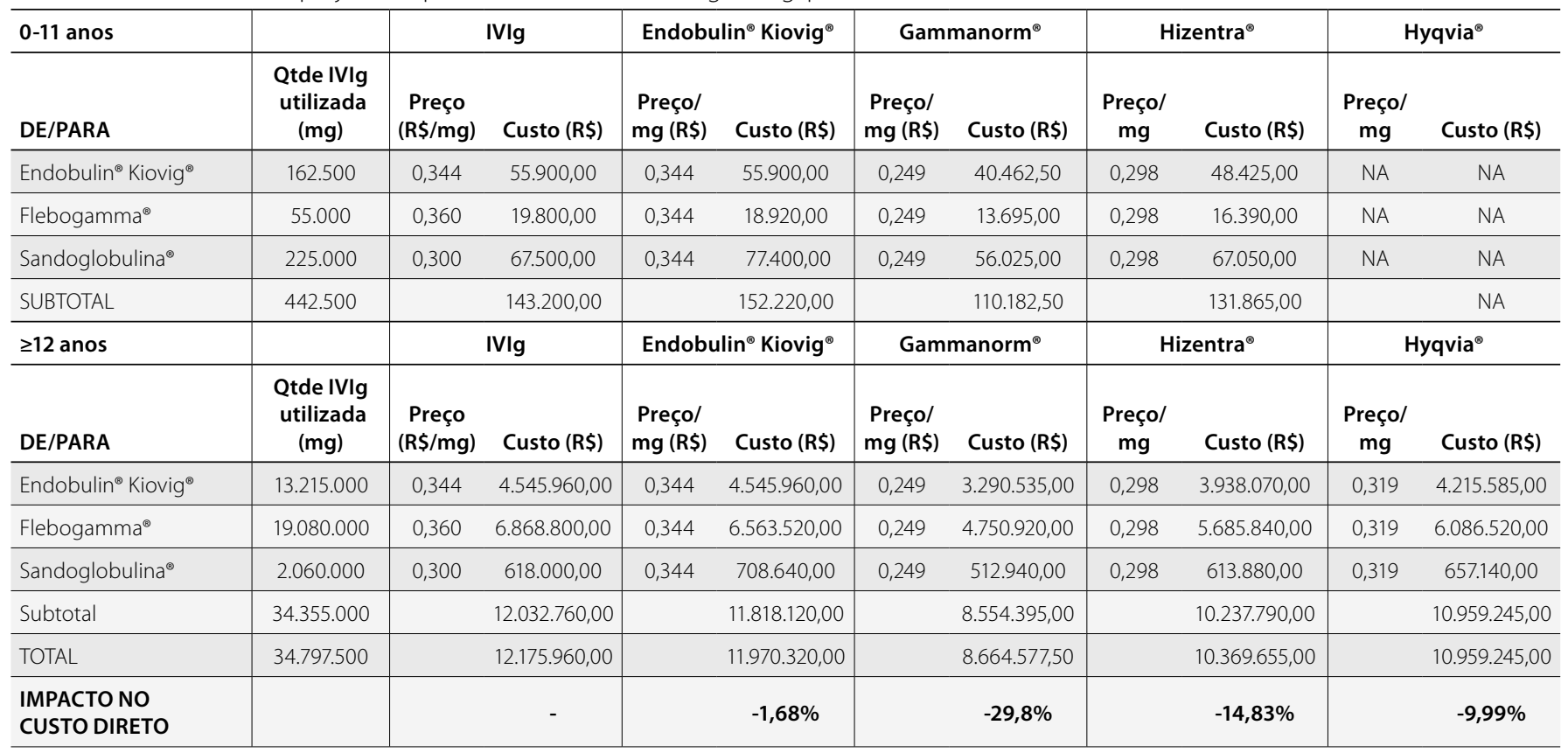

Fonte: Elaborada pelos autores (2020). Observação: Pacientes com Hyqvia com idade de 0 a 11, mantém o custo IVlg, pois não podem trocar para Hyqvia (indicação na bula para idade $\geq 12$ anos). Legenda - mg: miligramas.

mg) e também para o Endobulin Kiovig॰ (EV) vs. [Endobulin Kiovig (SC) R\$ 0,000/mg, Gammanorm: - R\$ 0,095/mg, Hizentra: - $R \$$ 0,046/mg e Hyqvia: : -R\$ 0,025/mg] e para a Sandoglobulina Privigen vs. (Endobulin Kiovig: $+R \$ 0,044 / \mathrm{mg}^{\circ}$ Gammanorm: - -R\$ 0,051/mg, Hizentra: :-R\$ 0,002/mg e Hyqviå: $+R \$ 0,019 / \mathrm{mg})$. A redução do custo direto após a substituição das IVIg pelas SClg foi de -R\$316.240,00 (-2,57\%) no cenário com Endobulin Kiovig', -R\$ 3.622.002,50 (-29,48\%) no cenário do Gammanorm; - -R\$ 1.916.925,00 (-15,60\%) no cenário do Hizentra e -R\$ 1.181.935,00 (-9,62\%) no cenário do Hyqvia.

Além disso, os custos inerentes às taxas de infusão são menores para a aplicação subcutânea $(R \$ 9,00)$ em relação à intravenosa ( $R \$ 103,50)$, ou seja, uma diferença de $-R \$ 94,50$, portanto representam um impacto econômico nos custos indiretos do tratamento na ordem de -R\$ 79.312,50 no cenário do Endobulin Kiovig', -R\$ 79.312,50 no cenário do Gammanorm, - -R\$100.462,50 no cenário do Hizentra e e -R\$ 107.352,00 no cenário do Hyqvia”.

Assim, observa-se vantagem econômica relevante na comparação do custo total (direto e indireto) de tratamento entre as tecnologias IVIg e SClg avaliadas, podendo chegar a uma minimização de até 29,83\% a favor das SClg, sendo: $-\mathrm{R} \$ 37.012,50$ (-0,30\%) no cenário do Endobulin Kiovig, - $R \$ 3.701 .315,00$ (-29,83\%) no cenário do Gammanorm: -R\$ 2.017.387,50 
Tabela 3. Custos totais projetados para os cenários de tratamentos de IVIg e SClg, estratificados por especialidade médica, para o período anual

\begin{tabular}{|c|c|c|c|c|c|c|}
\hline Especialidade & Consumo (MG) & IVlg & Gammnorm ${ }^{\oplus}$ & Hizentra ${ }^{\circledast}$ & Hyqvia ${ }^{\circledast}$ & $\begin{array}{c}\text { Endobulin }^{\oplus} \\
\text { Kiovig }^{\circledR}\end{array}$ \\
\hline $\begin{array}{l}\text { Alergia e } \\
\text { Imunologia }\end{array}$ & 1.110 .000 & $R \$ 386.394,00$ & $\mathrm{R} \$ 277.974,00$ & $R \$ 331.572,00$ & $R \$ 354.486,00$ & $\mathrm{R} \$ 383.424,00$ \\
\hline Clínica Geral & 4.110 .000 & $R \$ 1.451 .971,50$ & $R \$ 1.029 .474,00$ & $\mathrm{R} \$ 1.227 .822,00$ & $\mathrm{R} \$ 1.312 .611,00$ & $R \$ 1.419 .924,00$ \\
\hline $\begin{array}{l}\text { Ginecologia e } \\
\text { Obstetrícia }\end{array}$ & 20.000 & $R \$ 6.983,50$ & $R \$ 5.016,00$ & $R \$ 5.978,00$ & $R \$ 6.389,00$ & $\mathrm{R} \$ 6.916,00$ \\
\hline Nefrologia & 570.000 & $\mathrm{R} \$ 198.046,50$ & $\mathrm{R} \$ 142.614,00$ & $\mathrm{R} \$ 170.202,00$ & $\mathrm{R} \$ 182.001,00$ & $R \$ 196.764,00$ \\
\hline Neurologia & 920.000 & $R \$ 320.413,00$ & $\mathrm{R} \$ 230.448,00$ & $\mathrm{R} \$ 274.884,00$ & $R \$ 293.822,00$ & $\mathrm{R} \$ 317.848,00$ \\
\hline Oncologia Clínica & 13.875 .000 & $\mathrm{R} \$ 5.016 .504,50$ & $R \$ 3.468 .087,00$ & $\mathrm{R} \$ 4.141 .356,00$ & $\mathrm{R} \$ 4.429 .428,00$ & $\mathrm{R} \$ 4.786 .212,00$ \\
\hline Total & 34.797 .500 & $\mathrm{R} \$ 12.408 .192,50$ & R\$ 8.706.877,50 & $\mathrm{R} \$ 10.390 .845,00$ & $\mathrm{R} \$ 11.118 .905,50$ & $\mathrm{R} \$ 12.012 .640,00$ \\
\hline
\end{tabular}

Fonte: Elaborada pelos autores (2020).

(-16,26\%) no cenário do Hizentra e -R\$ 1.289.287,00 (-10,39\%) no cenário do Hyqvia:

Quando realizada a simulação, o custo com imunoglobulinas, no cenário das IVlg: R\$ $12.408 .192,50$ e média de $R \$ 93.294,68( \pm R \$ 76.590,32)$ por paciente, projetado para as SClg, passam a ser de R\$12.012.640,00 no cenário Endobulin Kiovig', com média de $R \$ 90.320,60( \pm R \$ 73.108,34)$ por paciente; R\$ 8.706.877,50 no cenário Gammanorm, com média de $R \$ 65.465,24$ ( $\pm R \$ 52.969,78)$ por paciente; $R \$ 10.390 .805,00$ no cenário Hizentrå, com média de R\$78.126,35 ( \pm R\$ 63.264,28) por paciente; R\$ 11.118.905,50 no cenário Hyqvia, com média de $R \$ 83.600,79( \pm R \$ 67.601,56)$ por paciente (Tabela 4).

\section{Discussão}

Este é o primeiro estudo de custo-minimização da troca de IVlg para SClg na saúde suplementar brasileira, que tem sua relevância ressaltada pela escassez de recursos econômicos no setor e o elevado patamar de inflação constatado no mercado de saúde (Garcia et al., 2013).

Importante colocar em um estudo de custo-minimização os principais fatores determinantes na precificação dos produtos em análise. O primeiro ponto a ser apontado é o papel dos ór- gãos reguladores oficiais. A CMED foi criada em 2003 por meio da Lei no 10.742 /2003, que extinguiu o órgão regulatório antecessor, denominado Câmara de Medicamentos (CAMED) (Brasil. Presidência da República, 2011). A CMED tem como atribuições a) autorizar preços de entrada para medicamentos novos no mercado brasileiro e b) acompanhar o mercado e autorizar reajustes de preços para medicamentos já comercializados (Brasil. Presidência da República, 2011). Além desse ponto, existem variações nas tributações regionais, questões estratégicas das empresas produtoras e distribuidoras, além de negociações e interesses individuais de cada operadora de saúde.

A questão da redução de custos em saúde aumenta sua importância perante as estimativas de expectativa de vida da população. Entre 1940 e 2015, a expectativa de vida ao nascer no Brasil aumentou cerca de 30 anos: de 45,5 anos em 1940, para 75,5 anos em 2015. Na última pesquisa (2018), essa expectativa passou para 76,3 anos (IBGE, 2019). Projeções do IBGE estimam que essa expectativa alcance os 80 anos em 2042 e, em 2060, chegaria aos 81,2 anos, e nessa data os idosos representarão, aproximadamente, $33 \%$ de toda a população residente no país. Com o aumento da população idosa, os países serão obrigados a investir suas receitas de forma cada vez mais efetiva, a fim de proporcionar os cuidados de saúde necessá-

Tabela 4. Custos médios e totais individuais para as simulações entre os cenários das IVlg e das SClg, para o período anual

\begin{tabular}{|c|c|c|c|c|c|}
\hline Parâmetro & IVIg & Gammnorm $^{\circledR}$ & Hizentra ${ }^{\circledast}$ & Hyqvia $^{\circledR}$ & Endobulin $^{\circledast}$ Kiovig $^{\circledR}$ \\
\hline 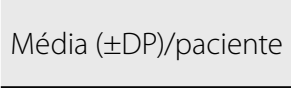 & $\begin{array}{c}R \$ 93.294,68 \\
( \pm R \$ 76.590,32)\end{array}$ & $\begin{array}{c}R \$ 65.465,24 \\
( \pm \operatorname{R} \$ 52.969,78)\end{array}$ & $\begin{array}{c}R \$ 78.126,35 \\
( \pm R \$ 63.264,28)\end{array}$ & $\begin{array}{c}R \$ 83.600,79 \\
( \pm R \$ 67.601,56)\end{array}$ & $\begin{array}{c}R \$ 90.320,60 \\
( \pm R \$ 73.108,34)\end{array}$ \\
\hline
\end{tabular}

Fonte: Elaborada pelos autores (2020). Legenda - DP: desvio-padrão; IVlg: imunglobulinas intravenosas. 
rios para toda a população. Desse modo, a redução de custos com imunoglobulinas constituem uma oportunidade de controle de custos, ao mesmo tempo em que a acessibilidade a esse tipo de terapêutica é assegurada (Puty \& Gentil, 2017; Shabaninejad et al., 2016).

Diante dos desafios iminentes e da necessidade de tratar um número cada vez maior de pessoas, vivendo por mais tempo, com múltiplas e complexas patologias, a expectativa é que os medicamentos biológicos saltem da participação atual, em torno de $20 \%$ do total de vendas do mercado farmacêutico global, para ocupar fatias crescentes na oferta de medicamentos. Também é inegável o crescimento sofrido por essa classe terapêutica: em 2010, as vendas mundiais de biológicos já se aproximavam dos 100 bilhões de dólares; em 2015, mais de 50\% das novas obtenções de autorização de introdução no mercado foram de medicamentos biológicos; estima-se um aumento para mais de 70\% em 2025 (Puty \& Gentil, 2017).

Ainda hoje não existe número suficiente de estudos independentes acerca da economia potencial provinda da entrada das SClg no mercado farmacêutico brasileiro. De maneira geral, as informações disponíveis na Alemanha (Högy et al., 2005), França (Beauté et al., 2010), Itália (Lazzaro et al., 2014), Japão (Igarashi et al., 2014) e Suíça (Pollock \& Meckley, 2018) apontam para uma diferença no custo global do tratamento entre IVIg e SClg de respectivamente: valor não encontrado; -5,97\%; -2,67\%; valor não encontrado; e -4,00\%. Esses dados corroboram os encontrados no presente estudo, no qual se identificou uma redução nos custos, após permuta entre as drogas, de até 29,8\%.

A introdução de SClg na listagem de medicamentos aprovados pelas operadoras permitirá uma melhora da qualidade de vida dos pacientes (Hadden \& Marreno, 2015; Igarashi et al., 2014), além da economia de valores consideráveis, para o sistema de saúde privado, independentemente da sua inclusão no Rol. Apesar de não ser alvo deste estudo, provavelmente o sistema público de saúde também pode se beneficiar dos resultados obtidos.

Por se tratar de produtos avaliados e aprovados pelas mesmas autoridades científicas que anteriormente aprovaram as IVIg, a utilização de SClg representa a possibilidade de aquisição de medicamentos com um preço mais acessível para o tratamento de doenças potencialmente fatais ou incapacitantes (Ebbers et al., 2012). Em contrapartida, apesar de ser uma alternativa menos onerosa e terapeuticamente equivalente a IVlg, é perceptível que a classe médica ainda apresenta dúvidas acerca das SClg e continua relutante em prescrevê-las com base no argumento da contenção de custos (Puty \& Gentil, 2017). Salienta-se que o custo dos medicamentos é um dos fatores que mais contribui para a não adesão ou interrupção das terapêuticas (Ebbers et al., 2012). Internacionalmente, percebem-se essas repercussões com maior expressão em países com baixos rendimentos.
O alto consumo e consequente alto custo na área de Oncologia Clínica e Hematologia/Hemoterapia (Tabela 3) traz uma reflexão sobre eventuais indicações dúbias do uso de IG nessas especialidades, o que levanta a necessidade de estudos com maiores níveis de evidência. As recomendações deveriam estar pautadas em estudos com níveis de recomendação mais elevados, como as revisões sistemáticas e metanálises de ensaios clínicos randomizados (nível de evidência l) ou de estudos de coorte (nível de evidência II). Esse fator já foi reforçado por uma revisão de literatura realizada pela American Academy of Allergy, Asthma \& Immunology (Perez et al., 2017), que sintetizou as várias indicações e suas respectivas probabilidades de real benefício ao paciente, bem como a categoria da evidência e a força da recomendação. Infelizmente, os dados foram oriundos, em sua grande parte, de estudos de menores níveis de evidências - III, IV e V -, os quais representam estudos de caso-controle e opinião de especialistas (CEBM, 2009).

Como limitações deste estudo, podemos destacar que não há garantia de que o período analisado seja representativo da realidade futura, por causa da obtenção transversal dos dados. Outro ponto a se destacar são os preços da CMED usados nos cálculos, os quais são os valores máximos de venda pelo fabricante e, assim, dependendo do desconto obtido em negociações, tanto das IVlg quanto das SClg, o resultado pode ser de impacto financeiro de minimização ou mesmo de adição. Além disso, vale ressaltar que os custos dependem de fatores locais e, portanto, podem dificultar a generalização para outros, no entanto, para evitar esse viés, os autores elaboraram um calculadora com análise de sensibilidade probabilística, na qual consideram variáveis como preço das IG, peso, posologia, taxas de aplicação, honorários, entre outros, gerando uma recomendação automática sobre quais trocas entre as IVIg e SClg (e vice-versa) são economicamente favoráveis para o cenário de cada instituição, o que viabiliza a análise personalizada para cada realidade da saúde suplementar brasileira; avaliações sobre segurança com dados de mundo real também fugiram do escopo deste estudo.

Embora ainda haja relutância para a prescrição das imunoglobulinas subcutâneas, estas possuem segurança e eficácia clínica consolidadas e estabelecidas. Assim, apresentam-se como uma alternativa terapêutica de menor custo para o sistema de saúde, atendendo ao aumento de demanda gerado pelo crescimento na incidência de pacientes com doenças imunológicas crônicas.

\section{Conclusões}

A escolha de uma SClg para o tratamento de doenças crônicas, ao invés da IVlg, traz benefícios econômicos à instituição pagadora e potencialmente ao paciente quando facilita o acesso à terapia e aumenta a qualidade de vida. 
Em conclusão, a análise de custo-minimização apresentada por este estudo aponta as SClg como uma alternativa terapêutica mais econômica e sustentável em relação ao tratamento com IVIg no cenário de redução de custos para a saúde suplementar no Brasil.

\section{Agradecimentos}

Os autores agradecem à Unimed Curitiba pelo apoio incondicional ao desenvolvimento de pesquisa e inovação.

\section{Referências bibliográficas}

Anvisa - Agência Nacional de Vigilância Sanitária. Câmara de Regulação do Mercado de Medicamentos (CMED). Available from: http://portal.anvisa. gov.br/listas-de-precos.

Beauté J, Levy P, Millet V, Debré M, Dudoit Y, Le Mignot L, et al.; French PID study group CEREDIH. Economic evaluation of immunoglobulin replacement in patients with primary antibody deficiencies. Clin Exp Immunol. 2010;160(2):240-5.

Bonilla FA, Khan DA, Ballas ZK, Chinen J, Frank MM, Hsu JT, et al.; Joint Task Force on Practice Parameters, representing the American Academy of Allergy, Asthma \& Immunology; the American College of Allergy, Asthma \& Immunology; and the Joint Council of Allergy, Asthma \& Immunology. Practice parameter for the diagnosis and management of primary immunodeficiency. J Allergy Clin Immunol. 2015;136(5):1186-205.e1-78.

Bousfiha AA, Jeddane L, Ailal F, Benhsaien I, Mahlaoui N, Casanova JL, et al. Primary immunodeficiency diseases worldwide: More common than generally thought. J Clin Immunol. 2013;33(1):1-7.

Brasil. Presidência da República. Lei no 12.527, de 18 de novembro de 2011. Regula o acesso a informações previsto no inciso XXXIII do art. 50, no inciso II do $\S 3^{\circ}$ do art. 37 e no $\S 2^{\circ}$ do art. 216 da Constituição Federal; altera a Lei no 8.112, de 11 de dezembro de 1990; revoga a Lei no 11.111, de 5 de maio de 2005, e dispositivos da Lei no 8.159, de 8 de janeiro de 1991; e dá outras providências.

CEBM - Centre for Evidence-Based Medicine. Oxford Centre for Evidencebased Medicine - Levels of Evidence (March 2009). Available from: http:// www.cebm.net/index.aspx?o=1025. Accessed on: Jan 8, 2020.

Chapel HM, Spickett GP, Ericson D, Engl W, Eibl MM, Bjorkander J. The comparison of the efficacy and safety of intravenous versus subcutaneous immunoglobulin replacement therapy. J Clin Immunol. 2000;20(2):94-100.

Drummond MF, Sculpher MJ, Torrance GW, O'Brien BJ, Stoddart GL. Methods for the Economic Evaluation of Health Care Programmes. Oxford: Oxford University Press; 2005.

Ebbers HC, Pieters T, Leufkens HG, Schellekens H. Effective pharmaceutical regulation needs alignment with doctors. Drug Discov Today. 2012;17(34):100-3.

Garcia LP, Sant'Anna AC, de Magalhães LCG, de Freitas LRS, Aurea AP. Gastos das famílias brasileiras com medicamentos segundo a renda familiar: Análise da Pesquisa de Orçamentos Familiares de 2002-2003 e de 20082009. Cad Saúde Pública. 2013;29(8):1605-16.
Gardulf A, Andersen V, Björkander J, Ericson D, Frøland SS, Gustafson R, et al. Subcutaneous immunoglobulin replacement in patients with primary antibody deficiencies: safety and costs. Lancet. 1995;345(8946):365-9.

Hadden RDM, Marreno F. Switch from intravenous to subcutaneous immunoglobulin in CIDP and MMN: Improved tolerability and patient satisfaction. Ther Adv Neurol Disord. 2015;8(1):14-9.

Högy B, Keinecke HO, Borte M. Pharmacoeconomic evaluation of immunoglobulin treatment in patients with antibody deficiencies from the perspective of the German statutory health insurance. Eur J Health Econ. 2005;6(1):24-9.

IBGE - Instituto Brasileiro de Geografia e Estatística. Em 2018, expectativa de vida era de 76,3 anos. 2019. Available from: https://agenciadenoticias. ibge.gov.br/agencia-sala-de-imprensa/2013-agencia-de-noticias/ releases/26104-em-2018-expectativa-de-vida-era-de-76-3-anos.

Igarashi A, Kanegane H, Kobayashi M, Miyawaki T, Tsutani K. Cost-minimization analysis of IgPro20, a subcutaneous immunoglobulin, in Japanese patients with primary immunodeficiency. Clin Ther. 2014;36(11):1616-24.

International Union of Immunological Societies Expert Committee on Primary Immunodeficiencies, Notarangelo LD, Fischer A, Geha RS, Casanova JL, Chapel H, Conley ME, et al. Primary immunodeficiencies: 2009 update. J Allergy Clin Immunol. 2009;124(6):1161-78.

Kashiura D, Souza P, Santos H, Nardi EP, Sá AB, Santos BR, et al. Modelo de impacto orçamentário do rituximabe subcutâneo comparado ao intravenoso no tratamento do linfoma não Hodgkin difuso de grandes células B, CD-20 positivo, no sistema de saúde suplementar brasileiro. J Bras Econ Saúde. 2018;10(3):255-61.

Lazzaro C, Lopiano L, Cocito D. Subcutaneous vs intravenous administration of immunoglobulin in chronic inflammatory demyelinating polyneuropathy: An Italian cost-minimization analysis. Neurol Sci. 2014;35(7):1023-34.

Perez EE, Orange JS, Bonilla F, Chinen J, Chinn IK, Dorsey M, et al. Update on the use of immunoglobulin in human disease: A review of evidence. J Allergy Clin Immunol. 2017;139(3S):S1-46.

Picard C, Al-Herz W, Bousfiha A, Casanova JL, Chatila T, Conley ME, et al. Primary Immunodeficiency Diseases: an Update on the Classification from the International Union of Immunological Societies Expert Committee for Primary Immunodeficiency 2015. J Clin Immunol. 2015;35(8):696-726.

Pollock RF, Meckley LM. An evaluation of the budget impact of a new $20 \%$ subcutaneous immunoglobulin (lg20Gly) for the management of primary immunodeficiency diseases in Switzerland. Clinicoecon Outcomes Res. 2018;10:223-9.

Puty CACB, Gentil DL, orgs. A Previdência Social em 2060: as inconsistências do modelo de projeção atuarial do governo brasileiro. Brasília: ANFIP; 2017. Disponível em: https://www.dieese.org.br/evento/2017/ aPrevidenciaSocialEm2016.pdf.

Shabaninejad H, Asgharzadeh A, Rezaei N, Rezapoor A. A Comparative Study of Intravenous Immunoglobulin and Subcutaneous Immunoglobulin in Adult Patients with Primary Immunodeficiency Diseases: A Systematic Review and Meta-Analysis. Expert Rev Clin Immunol. 2016;12(5):595-602.

Shabaninejad H, Asgharzadeh A, Rezapour A, Rezaei N. Cost-effectiveness analysis of subcutaneous immunoglobulin replacement therapy in Iranian patients with primary immunodeficiencies. Med J Islam Repub Iran. 2017;31:94. 